\title{
Spiral readouts for 4D flow MRI
}

\author{
Andreas Sigfridsson ${ }^{1,2^{*}}$, Sven Petersson ${ }^{1,2}$, Carl Johan Carlhall ${ }^{3,2}$, Tino Ebbers ${ }^{1,2}$ \\ From 15th Annual SCMR Scientific Sessions \\ Orlando, FL, USA. 2-5 February 2012
}

\section{Summary}

The feasibility of using spiral readouts to reduce the scan time of 4D flow MRI without sacrificing data quality was investigated.

\section{Background}

Time-resolved three-dimensional three-directional phase contrast MRI (4D flow) is being increasingly used to study the physiology and patophysiology of cardiovascular blood flow. However, the key limitation of existing implementations is the long scan times required. Spiral readouts are efficient and have appealing flow sensitivity properties. In this work, we investigate whether the scan time can be reduced by using spiral readout trajectories without reducing data quality.

\section{Methods}

A stack-of-spiral 4D flow pulse sequence was implemented on a 1.5T MRI scanner with identical spatiotemporal resolution as a conventional Cartesian pulse sequence $(2.8 \mathrm{~mm}$ isotropic voxel size, $48.8 \mathrm{~ms}$ temporal resolution). The thoracic aorta of ten healthy volunteers were imaged using both pulse sequences. The Cartesian acquisition used SENSE factor 2, whereas the spiral acquisition did not use parallel imaging. The spiral acquisition used water-selective excitation, TE/TR 3.5/ $12 \mathrm{~ms}$ with 12 spiral interleaves. Navigator gating with a window of $5 \mathrm{~mm}$ was used to suppress respiratory motion, and VENC was set to $1.5 \mathrm{~m} / \mathrm{s}$ for both techniques. The spiral acquisition was evaluated by comparing through-plane volume flow with the Cartesian technique. The ascending aorta was reformatted and segmented manually. Additionaly, the flow data quality was assessed by analyzing the number of pathlines that crossed the geometric boundaries of the aorta. Pathlines were emitted backwards from descending aorta in order

${ }^{1}$ Division of Cardiovascular Medicine, Department of Health Sciences, Linköping University, Linköping, Sweden

Full list of author information is available at the end of the article to avoid sensitivity to branching flow. Any errors in the flow data, including background phase errors, eddy currents, noise and blurring, will accumulate over the trajectory integration and trajectories that does not conform to the geometry thus indicate errors in the data.

\section{Results}

The scan times were $14 \mathrm{~m} 57 \mathrm{~s} \pm 4 \mathrm{~m} 36 \mathrm{~s}$ for the Cartesian acquisition and $6 \mathrm{~m} 12 \mathrm{~s} \pm 1 \mathrm{~m} 16 \mathrm{~s}$ for the spiral acquisition, including navigator gating. There was no difference between the cardiac output measured using the Cartesian acquisition compared to the spiral acquisition (6.2 \pm 1.0 vs $5.8 \pm 1.01 / \mathrm{min}$, NS). A Bland-Altman plot of the cardiac output is shown in Figure 1. The bias was -0.40 , and the limits of agreement were $\pm 1.13[1 / \mathrm{min}]$. The number of traces that were confined within the aortic boundaries during the pathline trace duration of 326 $\pm 40 \mathrm{~ms}$ was $51 \pm 16 \%$ for the Cartesian acquisition and 60 $\pm 7 \%$ for the spiral acquisition (NS). The pathlines of one of the volunteers are shown in Figure 2.

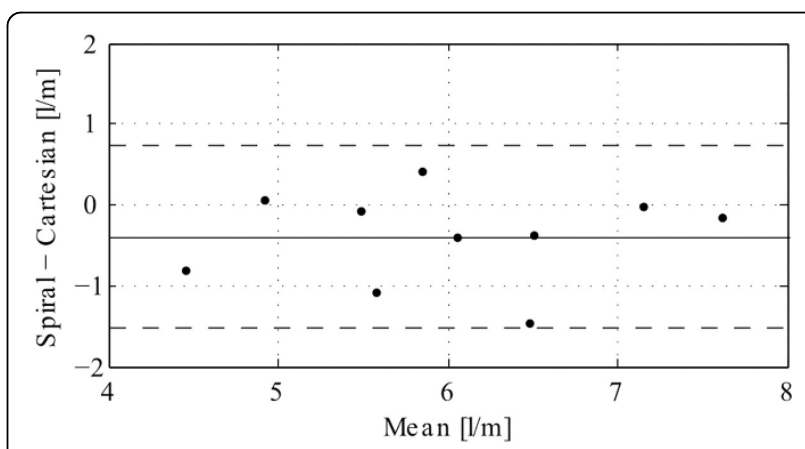

Figure 1 Bland-Altman analysis of the cardiac output measured using the spiral and the conventional Cartesian technique. The bias (solid line) was -0.40 , and the limits of agreement (dashed lines) were $\pm 1.13 \mathrm{l} / \mathrm{min}$. 


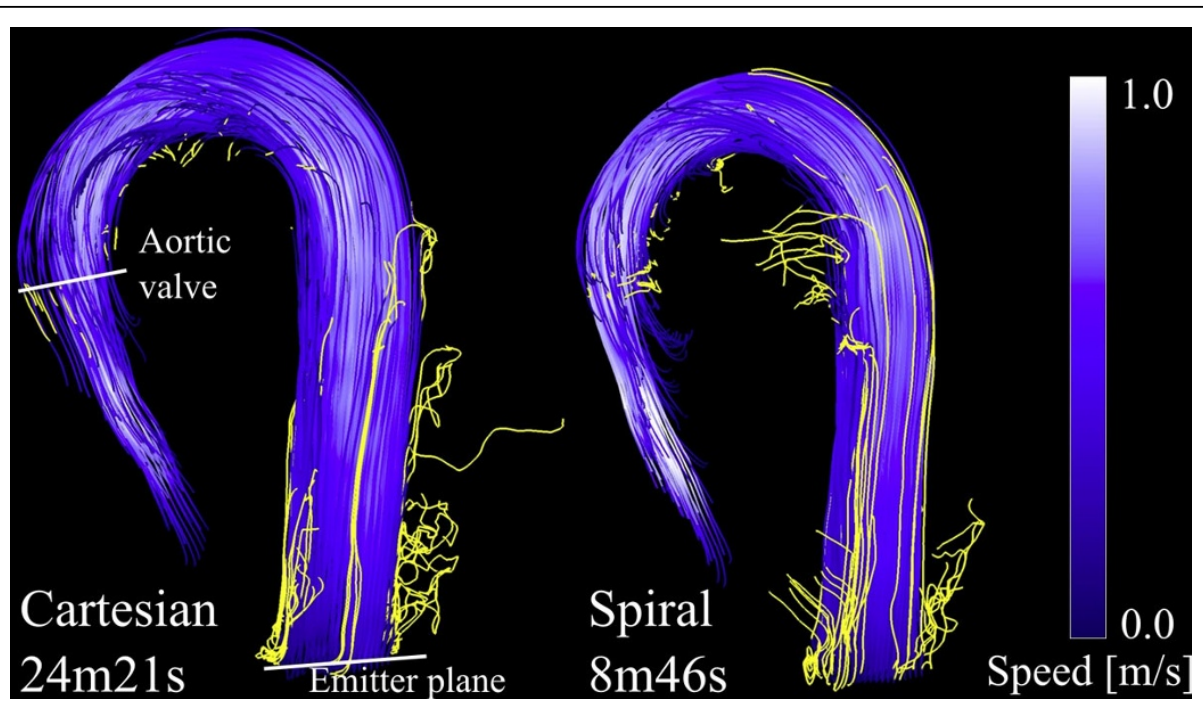

Figure 2 Pathlines generated from the data using both the conventional Cartesian technique and the spiral technique. The pathlines were traced backwards starting from the descending aorta, in order to avoid sensitivity to branching flow. Wall clock scan times (including the navigator efficiency) are indicated for this volunteer. Pathlines that leave the geometrical boundaries of the aorta (yellow) are detected and used as a measure for data fidelity to compare the two methods.

\section{Conclusions}

Using spiral readouts, the scan times could be reduced more than two-fold without sacrificing data quality. The spiral acquisition used in this work did not yet include parallel imaging, which could be used to further reduce the scan time. The shorter scan time may open up for broader use of 4D flow MRI for the assessment of cardiovascular flow in health and disease.

\section{Funding}

The Swedish Research Council, and the Swedish HeartLung Foundation.

\section{Author details}

${ }^{1}$ Division of Cardiovascular Medicine, Department of Health Sciences,

Linköping University, Linköping, Sweden. ${ }^{2}$ Center for Medical Image Science and Visualization (CMIV), Linköping University, Linköping, Sweden. ${ }^{3}$ Clinical

Physiology, Linköping University Hospital, Linköping, Sweden.

Published: 1 February 2012

\section{doi:10.1186/1532-429X-14-S1-W31}

Cite this article as: Sigfridsson et al:: Spiral readouts for 4D flow MRI.

Journal of Cardiovascular Magnetic Resonance 2012 14(Suppl 1):W31.
Submit your next manuscript to BioMed Central and take full advantage of:

- Convenient online submission

- Thorough peer review

- No space constraints or color figure charges

- Immediate publication on acceptance

- Inclusion in PubMed, CAS, Scopus and Google Scholar

- Research which is freely available for redistribution
Biomed Central 